\title{
Fully Decoupled Reliability-Based Optimization of Linear Structures Subject to Gaussian Dynamic Loading Considering Discrete Design 2 Variables
}

\author{
Matthias G. R. Faes ${ }^{a, *}$, Marcos A. Valdebenito ${ }^{\mathrm{b}}$ \\ ${ }^{a}$ KU Leuven, Department of Mechanical Engineering, Technology campus De Nayer, Jan De Nayerlaan 5, $\quad 5$ \\ St.-Katelijne-Waver, Belgium 6 \\ ${ }^{b}$ Faculty of Engineering and Sciences, Universidad Adolfo Ibáñez, Av. Padre Hurtado 750, 2562340 Viña del 7 \\ Mar, Chile
}

\begin{abstract}
Reliability-based optimization (RBO) offers the possibility of finding an optimal design for a 10 system according to a prescribed criterion while explicitly taking into account the effects of un- 11 certainty. However, due to the necessity of solving simultaneously a reliability problem nested in 12 an optimization procedure, the corresponding computational cost is usually high, impeding the 13 applicability of the methods. This computational cost is even enlarged further when one or sev- ${ }_{14}$ eral design variables must belong to a discrete set, due to the requirement of resorting to integer 15 programming optimization algorithms. To alleviate this issue, this contribution proposes a fully 16 decoupled approach for a specific class of problems, namely minimization of the failure probability 17 of a linear system subjected to an uncertain dynamic load of the Gaussian type, under the addi- ${ }_{18}$ tional constraint that the design variables are integer-valued. Specifically, by using the operator 19 norm framework, as developed by the authors in previous work, this paper shows that by reducing 20 the RBO problem with discrete design variables to the solution of a single deterministic optimiza- $\quad{ }^{21}$ tion problem followed by a single reliability analysis, a large gain in numerical efficiency can be 22 obtained without compromising the accuracy of the resulting optimal design. The application and 23 capabilities of the proposed approach are illustrated by means of two examples. 24 Keywords: Linear Structure, Gaussian loading, First excursion probability, Decoupling, $\quad 25$ Operator norm
\end{abstract}

*E-mail: matthias.faes@kuleuven.be 
- Optimal design under uncertainty considering discrete design variables is addressed.

- Optimization and reliability analyses are fully decoupled.

- Application of standard integer programming algorithms becomes feasible.

\section{Introduction}

Reliability-based optimization (RBO) offers a convenient framework for decision making in struc- 32 tural and mechanical engineering, as it allows exploring different design solutions and then, select- $\quad 33$ ing the best one according to a prescribed criterion [1]. Within such process, different sources of ${ }_{34}$ uncertainty that affect structural performance are described by means of probability theory and 35 synthesized in terms of a probability of occurrence. In this way, it is possible to determine design 36 solutions which are cost efficient, as available resources are used in an optimal way while ensuring $\quad 37$ an appropriate level of structural safety. RBO offers several advantages when compared to traditional deterministic design procedures. 39 Nonetheless, its practical application is usually quite challenging from a numerical viewpoint, 40 as it involves a double-loop approach [2]. That is, different design solutions must be explored ${ }_{41}$ and for each of them, it is necessary to perform uncertainty quantification considering different ${ }_{42}$ realizations of the uncertain parameters affecting performance. In view of this challenge, sev- ${ }^{43}$ eral implementations of RBO that offer improved numerical efficiency have been proposed, which ${ }_{44}^{44}$ involve, for example, efficient integration of algorithms for optimization and reliability analysis 45 (see, e.g. $[3,4,5,6,7]$ ), application of approximation concepts and surrogate models (see, e.g. 46 $[8,9,10,11])$ or specialized programming techniques that avoid double-loop implementation (see, $\quad 47$ e.g. $[12,13,14,15,16])$. For a more detailed overview on approaches for RBO, it is referred to, 48 e.g. $[1,2,17,18]$.

Most of the approaches developed for RBO consider design variables that can be modeled as 50 real numbers lying within a prescribed interval. Nonetheless, in several practical cases, design ${ }_{51}$ variables must be chosen from an existing inventory offered by a manufacturer. For example, 52 when designing steel structures, elements such as beams or columns must be selected out of a ${ }_{53}$ catalog of available profiles. Such issue posses a major challenge for RBO, as the associated op- ${ }^{54}$ timization problem must be solved resorting to algorithms for integer programming, which are 55 
generally much more demanding than their continuous counterparts [19,20], and in some cases 56 even NP-hard. Hence, specialized RBO strategies have been developed for solving problems in- 57 volving discrete design variables. These strategies can be broadly classified into three classes. The 58 first one involves direct coupling of algorithms for discrete optimization and reliability analysis, 59 as proposed in, e.g. [21, 22, 23, 24, 25, 26]. The second class of approaches consists of construct- 60 ing a surrogate model for reliability, thus rendering the application of the optimization algorithm 61 trivial, see e.g. $[10,27,28]$. The third class of approaches consists of decoupling the discrete 62 optimization and reliability analysis steps by creating local approximate representations of the 63 reliability, e.g. $[29,30,31,32,33,34]$. As an additional remark, it should be noted that RBO ${ }_{64}$ formulations addressing both discrete and continuous design variables (which are termed as mixed 65 discrete-continuous) also constitute another important class of problems, see e.g. [31, 35, 36]. ${ }_{66}$ Mixed discrete-continuous RBO problems can be found, for example, in structural engineering 67 [35], where one may consider discrete variables for selecting commercially available cross sections $\quad 68$ and continuous variables for dimensions that define the shape of the structure. 69 This contribution proposes an approach for RBO considering discrete design variables for a spe- 70 cific class of problems, that is, minimization of the first excursion probability of linear structures $\quad 71$ subject to stochastic Gaussian loading. The proposed approach exploits the specific nature of 72 the problem considered and departs from other developments in the literature as it is formulated 73 in a fully decoupled fashion. This means that the whole RBO problem is addressed by solving 74 a single deterministic discrete optimization problem followed by a single reliability estimation. 75 Such formulation is quite advantageous from a numerical viewpoint, as it effectively breaks the 76 double-loop that arises in classical approaches for RBO. Furthermore, as the optimization problem 77 to be solved is of deterministic nature, there is no need to deal with sampling estimators, which 78 may become troublesome, see e.g. [6]. The theoretical basis of this fully decoupled formulation 79 lies on the application of the operator norm theorem [37], which has already been explored by 80 the authors within the context of imprecise reliability analysis [38] and optimization under uncer- 81 tainty considering continuous design variables [39]. For practical implementation of the proposed 82 approach, discrete optimization is carried out by means of Genetic Algorithms (see, e.g. [40, 41]) 83 while reliability analysis is conducted by means of Directional Importance Sampling [42, 43]. 84 The rest of this contribution is organized as follows. Section 2 provides a detailed formulation of 85 the type of problems considered in this contribution. Then, Section 3 presents the fully decoupled 86 
strategy to solve the RBO problem. The practical application of this strategy is analyzed by 87 means of examples in Section 4. The paper concludes with discussions and outlook for future 88 research in Section 5.

\section{Formulation of the problem}

\subsection{General remarks}

This section formulates the class of problems considered in this contribution, namely the min- 92 imization of the first excursion probability of a linear structural system subject to stochastic 93 loading with design variables of discrete type. The discussion starts with the representation of the 94 stochastic loading in Section 2.2. The characterization of the structural response is presented in 95 Section 2.3. Then, the first excursion probability problem as well as the RBO problem involving 96 discrete design variables are formulated in Sections 2.4 and 2.5, respectively.

\subsection{Stochastic loading}

It is assumed that dynamic loading $f$ acting over the structural system can be characterized as 99 a Gaussian stochastic process. Such model has been considered in several practical applications, 100 see e.g. $[44,45]$. The stochastic process possesses a duration $T$ and is represented at discrete time ${ }_{101}$ instants $t_{k}=(k-1) \Delta t, k=1, \ldots, n_{T}$, where $\Delta t$ is the time step and $n_{T}$ is the total number of $\quad 102$ time instants considered. The covariance of the discretized Gaussian process is denoted as matrix ${ }_{103}$ $\Gamma$ of dimension $n_{T} \times n_{T}$ and it is assumed for the sake of simplicity that the mean of the process ${ }_{104}$ is zero. Following the above assumptions and applying the Karhunen-Loève expansion (see, e.g. 105 [46]), the stochastic loading is described as:

$$
\boldsymbol{f}(\boldsymbol{z})=\boldsymbol{\Psi} \Lambda^{1 / 2} \boldsymbol{z}
$$

where $\boldsymbol{f}$ is a realization of the stochastic loading, which is a $n_{T} \times 1$ vector, whose $k$-th component ${ }_{107}$ $f_{k}$ represents the loading at time $t_{k} ; \boldsymbol{\Psi}$ is a matrix of dimension $n_{T} \times n_{K L}$ containing the first ${ }_{108}$ $n_{K L}$ eigenvectors of the covariance matrix $\boldsymbol{\Gamma} ; \boldsymbol{\Lambda}$ is a matrix whose diagonal contains the first $n_{K L} \quad{ }^{109}$ eigenvalues of the covariance matrix $\Gamma ; n_{K L}$ is the number of terms retained for the Karhunen- ${ }_{110}$ Loève expansion $\left(n_{K L} \leq n_{T}\right.$, see, e.g. [46]); and $\boldsymbol{z}$ is a realization of a standard Gaussian random 111 variable vector $\boldsymbol{Z}$ of dimension $n_{K L} \times 1$ and whose probability density function is denoted as 112 $p_{\boldsymbol{Z}}(\boldsymbol{z})$. 
Gaussian stochastic processes offer the means for modeling uncertain dynamic loading in a number $\quad{ }_{114}$ of problems, see e.g. [47]. Naturally, for some other problems, non-Gaussian stochastic process 115 may be required. However, the latter is outside the scope of this contribution. 116

\subsection{Structural model and its response}

It is considered that the behavior of the structural system due to the stochastic loading can be 118 modeled as linear elastic with classical damping. The associated equation of motion is [48, 49]: 119

$$
\boldsymbol{M}(\boldsymbol{y}) \ddot{\boldsymbol{x}}(t, \boldsymbol{y}, \boldsymbol{z})+\boldsymbol{C}(\boldsymbol{y}) \dot{\boldsymbol{x}}(t, \boldsymbol{y}, \boldsymbol{z})+\boldsymbol{K}(\boldsymbol{y}) \boldsymbol{x}(t, \boldsymbol{y}, \boldsymbol{z})=\boldsymbol{\rho} f(t, \boldsymbol{z})
$$

where $t$ denotes time; $\boldsymbol{M}, \boldsymbol{C}$ and $\boldsymbol{K}$ are the mass, damping and stiffness matrices of dimension 120 $n_{D} \times n_{D}$ each; $n_{D}$ is the number of degrees-of-freedom of the structural model; $\boldsymbol{y}$ denotes a 121 vector of design variables, which may affect the mass, damping and stiffness matrices; $\boldsymbol{\rho}$ is a 122 vector of dimension $n_{D} \times 1$ coupling the stochastic load $f(t, \boldsymbol{z})$ with the degrees-of-freedom of the $\quad 123$ structure; and where $\ddot{\boldsymbol{x}}, \dot{\boldsymbol{x}}$ and $\boldsymbol{x}$ denote the acceleration, velocity and displacement vectors, each 124 of dimension $n_{D} \times 1$. 125 Practical design situations demand monitoring $n_{R}$ responses of interest of the structural system. $\quad 126$ These responses are denoted in the following as $\eta_{i}(t), i=1, \ldots, n_{R}$ and due to the linearity of ${ }_{127}$ the structure, are calculated by means of a convolution integral, that is:

$$
\eta_{i}(t, \boldsymbol{y}, \boldsymbol{z})=\int_{0}^{t} h_{i}(\boldsymbol{y}, t-\tau) f(\tau, \boldsymbol{z}) d t, i=1, \ldots, n_{R}
$$

where $h_{i}$ denotes the associated unit impulse response function (see Appendix A). In view of ${ }_{129}$ the discrete time representation considered in Section 2.2, the different responses of interest at 130 different time instants can be calculated as:

$$
\boldsymbol{\eta}_{i}(\boldsymbol{y}, \boldsymbol{z})=\boldsymbol{A}_{i}(\boldsymbol{y}) \boldsymbol{z}, i=1, \ldots, n_{R}
$$

where $\boldsymbol{\eta}_{i}$ is a vector of dimension $n_{T} \times 1$ containing the discrete-time representation of the $i$-th $\quad 132$ response along the duration $T$ of the stochastic loading; and $\boldsymbol{A}_{i}$ is a matrix of dimension $n_{T} \times n_{K L} \quad{ }_{133}$ whose $k$-th row contains the discrete time representation of the convolution integral in eq. (3), as 134 discussed in detail in Appendix B. 135 Eq. (4) provides a compact expression for calculating the $i$-th response of interest in time using 136 
matrix and vector operations. From this equation, it is important to note that the response is a 137 function of both the properties of the structural system and in particular, its design variables $\boldsymbol{y}, \quad 138$ as well as a function of the stochastic load, represented through $\boldsymbol{z}$. In other words, the responses 139 of interest of the system are uncertain because they depend on $\boldsymbol{z}$, but can also be modified by 140 $\begin{array}{ll}\text { changing the design variables } \boldsymbol{y} . & 141\end{array}$

\subsection{First excursion probability 142}

For practical design purposes, it is desirable that absolute value of the responses of interest remain 143 below acceptable threshold levels $b_{i}, i=1, \ldots, n_{R}$ within the duration $T$ of the stochastic load. 144 For verifying such condition, it is useful to define the so-called normalized response function $r(\boldsymbol{y}, \boldsymbol{z}) \quad 145$ [50], which is defined as:

$$
r(\boldsymbol{y}, \boldsymbol{z})=\|\boldsymbol{A}(\boldsymbol{y}) \boldsymbol{z}\|_{\infty}
$$

where $\|\cdot\|_{\infty}$ denotes infinity norm; and $\boldsymbol{A}$ is a matrix that collects all matrices $\boldsymbol{A}_{i}$ associated with $\quad{ }_{147}$ the calculation of the $i$-th response (see eq. (4)), normalized by their respective threshold levels. 148 Under the assumption that the thresholds fulfill the condition $b_{i}>0, i=1, \ldots, n_{R}$, matrix $\boldsymbol{A}$ is $\quad 149$ defined as:

$$
\boldsymbol{A}(\boldsymbol{y})=\left[\begin{array}{c}
b_{1}^{-1} \boldsymbol{A}_{1}(\boldsymbol{y}) \\
\vdots \\
b_{n_{R}}^{-1} \boldsymbol{A}_{n_{R}}(\boldsymbol{y})
\end{array}\right]
$$

and its dimension is $\left(n_{R} n_{T}\right) \times n_{K L}$. The definition of matrix $\boldsymbol{A}$ as shown in eq. (6) ensures that 151 all responses are calculated in a dimensionless fashion. In this way, it is possible to compare 152 on a similar basis responses involving displacements, accelerations, etc. In addition, the infinity 153 norm in eq. (5) allows retrieving the maximum normalized response. Naturally, in case $r<1, \quad 154$ all $n_{R}$ responses of interest of the structure remain below its prescribed threshold throughout 155 the duration of the stochastic load. On the contrary, in case $r \geq 1$, one or more responses of ${ }_{156}$ interest exceed their prescribed threshold at one or more time instants and the structure exhibits $\quad 157$ undesirable behavior. The chances that such undesirable behavior occurs within the duration 158 of the stochastic loading is termed as the first excursion probability (see, e.g. [47]), which is 159 
calculated by means of the following classical integral:

$$
p_{F}(\boldsymbol{y})=\int_{\boldsymbol{z} \in \mathbb{R}^{n} K L} I_{F}(\boldsymbol{y}, \boldsymbol{z}) p_{\boldsymbol{Z}}(\boldsymbol{z}) d \boldsymbol{z}
$$

where $p_{F}$ denotes the first excursion probability (or simply, the failure probability); and $I_{F}(\cdot, \cdot)$ is 161 the indicator function, which is equal to one in case $r(\boldsymbol{y}, \boldsymbol{z}) \geq 1$ and zero, otherwise. Note that 162 the first excursion probability as shown above corresponds to a series system formulation. Such ${ }_{163}$ probability is most useful for verifying, for example, serviceability conditions, as considered in this 164 contribution. However, for analysis of redundant systems and collapse, such may not be the case. 165 The practical estimation of the failure probability as shown in eq. (7) is far from trivial for cases of 166 practical interest. This stems out of two issues. First, the number of terms $n_{K L}$ to be retained for ${ }_{167}$ the Karhunen-Loève representation may be large (in the order of hundreds or thousands). Second, 168 the normalized response function $r$ (which is required for calculating the indicator function) is not 169 known in closed form, and must be calculated for a specific structural setting (given by the design 170 variable vector $\boldsymbol{y}$ ) and a specific realization of the stochastic loading (given by the realization $\boldsymbol{z}$ ). 171 Thus, these two issues favor the application of advanced simulation methods for estimating the 172 failure probability [51].

\subsection{Reliability-based optimization considering discrete design variables}

The structure of eq. (2) indicates that the structural response depends on the design variable vector 175

$\boldsymbol{y}$. This can be understood on physical grounds considering that the components of this design 176 variable vector represent, for example, the dimensions of cross section of structural members. 177 Thus, it is evident that by modifying cross sections, it is possible to alter the structural response 178 (assuming a fixed stochastic load $\boldsymbol{z}$ ) and in turn, the normalized response function $r$. Hence, the 179 failure probability itself becomes a function of these design variables, as stated in eq. (7). $\quad 180$ The fact that the value of the failure probability can be changed depending on the value of $\boldsymbol{y} \quad 181$ opens the possibility for performing structural design within the framework of optimization. In 182 such context, several different formulations for the optimization problem can be devised [18], for 183 example: minimization of structural weight subject to probability constraints (see, e.g. [52]); 184 minimization of failure probability under a set of available resources (see, e.g. [5, 53]); or risk- ${ }_{185}$ optimization taking into account the costs of construction, failure and repair (see, e.g. [54]). For 186 a comparison of such formulations, it is referred to, e.g. [55]. For the particular case of this 187 
contribution, the focus is on problems involving the minimization of the failure probability given 188 a set of available resources. Moreover, it is assumed that the design variables are of discrete type. 189 Such selection reflects the case where there is a finite number of choices for design variables due to, 190 e.g. fabrication constraints. The class of problems considered herein is quite useful when different 191 types of failure modes affect the reliability of a structural system. In this sense, minimization 192 of the failure probability constrained on available resources allows determining solutions which 193 may represent a trade-off between those failure modes [5, 53]. Mathematically, the problem to be 194 solved is formulated as follows.

$$
\begin{aligned}
& \min _{\boldsymbol{y}} p_{F}(\boldsymbol{y}) \\
& \text { subject to } \\
& c_{l}(\boldsymbol{y}) \leq 0, l=1, \ldots, n_{C} \\
& y_{j} \in \Omega_{j}=\left\{y_{j, 1}, y_{j, 2}, \ldots, y_{j, e_{j}}\right\}, j=1, \ldots, n_{y}
\end{aligned}
$$

where $c_{l}(\cdot)$ represents a deterministic constraint; $y_{j}$ is the $j$-th element of $\boldsymbol{y}$; $n_{y}$ denotes the 196 dimension of the design variable vector; $\Omega_{j}$ denotes the set of available discrete values that the ${ }_{197}$ design variable $y_{j}$ may assume; $e_{j}$ is the number of elements contained in set $\Omega_{j}$; and $\Omega$ is the set 198 of all values that $\boldsymbol{y}$ may assume, which is equal to the Cartesian product $\Omega_{1} \times \ldots \times \Omega_{n_{y}}$. Within ${ }_{199}$ the context of this contribution, it is noted that the design variable $y_{j}$ may represent directly a 200 certain structural property, for example, the thickness of an element, which assumes specific values 201 due to fabrication constraints. Or in the contrary, the design variables $y_{j}$ may just be an integer ${ }_{202}$ number that points out, for example, to a database of existing steel profiles. 203 The challenge with the solution of the optimal design problem in eq. (8) is threefold. First, the 204 solution of an optimization problem involving discrete design variables is usually quite demanding 205 on its own, as a large number of different structural configurations (that is, different values for 206 the design variables) must be considered. This implies in turn carrying out several structural 207 analyses. Second, the calculation of the failure probability associated with a given design vector by 208 means of simulation (as discussed in Section 2.4) demands performing repeated structural analyses 209 for different realizations of the stochastic loading vector $\boldsymbol{z}$. Third, the calculation of the failure ${ }_{210}$ probability must be carried within the optimization algorithm, leading to the so-called double loop ${ }^{211}$ implementation. These three issues impose a numerical burden which can be prohibitive, even ${ }_{212}$ 
for relatively simple models. Hence, in the following, an approach for solving the optimization 213 problem in eq. (8) is developed, which can break the double loop by applying the operator norm ${ }^{214}$ theorem.

\section{Fully Decoupled Reliability-based Optimization}

\subsection{Operator norm theorem as a means for decoupling}

Consider a matrix $\boldsymbol{B}$ of dimension $n_{1} \times n_{2}$ and a vector $\boldsymbol{\xi}$ of dimension $n_{2} \times 1$. The operator ${ }_{218}$ norm theorem (see, e.g. [37]) allows answering the following question: what is the largest value $c \quad{ }_{219}$ by which matrix $\boldsymbol{B}$ stretches $\boldsymbol{\xi}$ ? Such question is posed in terms of a $p^{(1)}$-norm applied over the ${ }_{220}$ vector $\boldsymbol{B} \boldsymbol{\xi}$ (that is, the stretched vector) with respect to another $p^{(2)}$-norm applied over vector $\boldsymbol{\xi}{ }^{221}$ (that is, the vector before stretching). Mathematically, the operator norm theorem is expressed ${ }_{222}$ as:

$$
\|\boldsymbol{B}\|_{p^{(1)}, p^{(2)}}=\inf \left\{c \geq 0:\|\boldsymbol{B} \boldsymbol{\xi}\|_{p^{(1)}} \leq c\|\boldsymbol{\xi}\|_{p^{(2)}}\right\}
$$

where $\inf \{\cdot\}$ denotes infimum; $\|\cdot\|_{p^{(1)}, p^{(2)}}$ denotes operator norm with respect to $p$-norms $p^{(1)}{ }_{224}$ and $p^{(2)} ;\|\cdot\|_{p}$ denotes $p$-norm; and where $c$ is a number that represents the maximum possible ${ }_{225}$ stretch. A comparison of eq. (9) with eq. (5) allows establishing the following connection between 226 the operator norm theorem and the calculation of the normalized response function: matrix ${ }_{227}$ $\boldsymbol{B}$ corresponds to $\boldsymbol{A}$; vector $\boldsymbol{\xi}$ corresponds to $\boldsymbol{z}$; and $p^{(1)} \rightarrow \infty$. In addition and in order to ${ }_{228}$ apply the operator norm theorem as cast in eq. (9), it is assumed that $p^{(2)}=2$, as this can be ${ }^{229}$ loosely associated with the energy content of the stochastic load [38]. Thus, under the previous ${ }^{230}$ observations and assumption, the following operator norm associated with matrix $\boldsymbol{A}$ is formulated. ${ }^{231}$

$$
\|\boldsymbol{A}(\boldsymbol{y})\|_{\infty, 2}=\inf \left\{c(\boldsymbol{y}) \geq 0:\|\boldsymbol{A}(\boldsymbol{y}) \boldsymbol{z}\|_{\infty} \leq c(\boldsymbol{y})\|\boldsymbol{z}\|_{2}\right\}
$$

The expression above implies that the stochastic load vector can be stretched by matrix $\boldsymbol{A}$ by ${ }^{232}$ factor $c$ at most. As discussed in [37], the solution to the operator norm problem in eq. (10) is 233 equal to the row of matrix $\boldsymbol{A}$ with largest Euclidean norm, that is:

$$
\|\boldsymbol{A}(\boldsymbol{y})\|_{\infty, 2}=\max _{\substack{i=1, \ldots, n_{R} \\ k=1, \ldots, n_{T}}}\left(\sqrt{\boldsymbol{a}_{i_{k}} \boldsymbol{a}_{i_{k}}^{T}}\right)
$$


where $\boldsymbol{a}_{i_{k}}$ represents the row of matrix $\boldsymbol{A}$ associated with the calculation of the $i$-th response at ${ }^{235}$ time instant $t_{k}$ (see Appendix B for more details). 236 An examination of eq. (10) reveals that as the stretched stochastic load is actually the normalized ${ }_{237}$ response function (see eq. (5)), factor $c$ expresses the maximum amplification that the stochastic ${ }^{238}$ load may undergo when applied to the structure. Furthermore, as the normalized response function 239 governs the calculation of the failure probability (see eq. (7)), it is postulated that minimizing the 240 operator norm associated with $\|\boldsymbol{A}(\boldsymbol{y})\|_{\infty, 2}$ is equivalent to minimizing the failure probability; in ${ }^{241}$ other words, by minimizing the maximum stretching factor of the load, one is actually causing the 242 failure event to be less likely. Such reasoning, which combines physical and mathematical grounds, 243 means that the operator norm of matrix $\|\boldsymbol{A}(\boldsymbol{y})\|_{\infty, 2}$ offers a suitable proxy for determining the set ${ }_{244}$ of design variables which yields the minimum value of the failure probability as in eq. (8). The ${ }^{245}$ quality of this proxy has been verified and validated, e.g. for estimating imprecise probabilities in 246 [38]. Therefore, the RBO problem formulated in eq. (8) can be replaced by the following problem: ${ }^{247}$

$$
\begin{aligned}
& \underset{\boldsymbol{y}}{\min }\|\boldsymbol{A}(\boldsymbol{y})\|_{\infty, 2} \\
& \text { subject to } \\
& c_{l}(\boldsymbol{y}) \leq 0, l=1, \ldots, n_{C} \\
& y_{j} \in \Omega_{j}=\left\{y_{j, 1}, y_{j, 2}, \ldots, y_{j, e_{j}}\right\}, j=1, \ldots, n_{y}
\end{aligned}
$$

The advantage of the problem formulated above is that it does not involve the stochastic load, 248 as the operator norm $\|\boldsymbol{A}(\boldsymbol{y})\|_{\infty, 2}$ depends purely on the information contained in matrix $\boldsymbol{A}$ and 249 not on vector $\boldsymbol{z}$. In other words, the optimization problem in eq. (12) is actually a deterministic 250 optimization problem. Once the optimum $\boldsymbol{y}^{\star}$ has been found by solving eq. (12), it suffices to 251 carry out a single reliability analysis for that optimum in order to determine the minimum failure 252 probability $p_{F}\left(\boldsymbol{y}^{\star}\right)$ associated with the RBO problem in eq. (8). 253 The framework for determining the optimum design $\boldsymbol{y}^{\star}$ and its associated failure probability as ${ }^{254}$ described above has important implications from a practical viewpoint. First, the optimization 255 problem in eq. (12) is purely deterministic, which means that its solution does not involve any 256 double loop for conducting reliability assessment. This single fact can decrease numerical costs ${ }^{257}$ significantly. Furthermore, the optimization problem in eq. (12) can be solved by means of any 258 suitable algorithm. Once the optimal design has been found, reliability analysis is conducted a 259 
single time for that optimum. This means that the traditional double-loop encountered when 260 solving RBO problems (that is, reliability assessment nested within an optimization procedure) is 261 effectively broken and replaced by one deterministic optimization followed by a single reliability 262 analysis. Thus, the proposed approach possesses the potential for decreasing the numerical cost 263 associated with the solution of RBO problems significantly. 264 The approach for RBO as described above can be highly advantageous from a numerical viewpoint 265 due to its decoupled nature. In this sense, the operator norm theorem takes full advantage of the 266 structure of the problem under consideration, that is, minimization of the failure probability under $\quad 267$ certain constraints. However, the extension of the proposed approach to other classes of problems 268 which are relevant in RBO may not be straightforward. For example, the solution of problems 269 involving weight minimization under probability constraints or risk-based optimization [18, 55] 270 cannot be directly solved following the proposed approach. This stems out of the fact that the ${ }^{271}$ operator norm as considered in the paper does not provide information on the actual value of ${ }_{272}$ the failure probability itself but just about the set of design variables that minimizes the failure ${ }^{273}$ probability.

\subsection{Optimization considering discrete design variables}

The optimization problem in eq. (12) involves design variables that belong to a discrete set $\Omega$. 276 Such class of problems may not be amenable for classical gradient-based algorithms (see, e.g. [56]) 277 and hence, one must resort to alternatives algorithms such as branch-and-bound methods [57], 278 annealing methods [58] or evolutionary algorithms [40,59]. In this work, the optimization problem 279 involving discrete design variables is solved by means of Genetic Algorithms, as its application for 280 solving structural problems has been widely tested and validated in the literature, see e.g. [56]. 281 Genetic Algorithms (GA) correspond to a class of optimization algorithms which rely on analogies 282 to natural processes, that is, natural evolution. GA are one of three main streams of the so-called ${ }^{283}$ evolutionary algorithms for optimization, while the other two streams are Evolution Strategies and 284 Evolutionary Programming, respectively (see, e.g. [60]). GA closely resembles the evolution of 285 biological organisms, as it employs a population of possible solutions instead of a unique solution; 286 this is a distinctive characteristic, as many other algorithms (such as gradient-based optimization 287 algorithms) consider a single candidate solution. Each individual of the population of solutions 288 has associated a certain fitness, which is a metric on how good a solution is from the point of view 289 of the optimization problem. The individuals with highest fitness value can reproduce by means 290 
of specific operations (namely, crossover and mutation) in order to generate a new population. 291 This new population possesses properties of the individuals of highest fitness of the previous 292 population. In this way, by generating a number of populations, it is possible explore the space 293 of design variables in order to determine the most promising locations [61]. It should be noted 294 that GA is a gradient-free optimization algorithm. Moreover, design constraints can be included 295 resorting to penalization, as described in [62].

From a numerical viewpoint, the application of GA may be quite demanding, as a single population ${ }^{297}$ may involve several individuals, which evolve through several generations. In turn, the evaluation 298 of the fitness of each individual corresponds to calculating the operator norm associated with 299 matrix $\boldsymbol{A}$ as well as the deterministic constraints $c_{l}, l=1, \ldots, n_{C}$, as noted from eq. (12). While 300 numerical evaluation of the deterministic constraints is usually trivial, the computation of the 301 operator norm demands performing structural analysis (that is, eigenvalue/eigenvector analysis). 302

Eventually, the computation of the operator norm within the optimization problem in eq. (12) 303 could be sped up by exploiting the linearity of the structure by means of substructuring concepts 304 $[63,64]$. Nonetheless, such possibility is not further explored in this contribution, as the focus 305 is on the concept of decoupling and its practical application for RBO considering discrete design 306 variables.

\subsection{Estimation of first excursion probability}

After identifying the optimal design $\boldsymbol{y}^{\star}$ that solves eq. (12) by means of GA as described above, it is 309 necessary to estimate the failure probability associated with that design. Such task could be carried 310 out by applying, for example, Monte Carlo simulation (see, e.g. [65]), which would imply generat- 311 ing $N$ random samples $\boldsymbol{z}^{(l)}, l=1, \ldots, N$ that follow a standard Gaussian distribution and then, 312 evaluating the dynamic response (see eq. (5)) and the indicator function for each of those samples. 313 Thus, the sought failure probability would be estimated as $p_{F}\left(\boldsymbol{y}^{\star}\right) \approx \sum_{l=1}^{N} I_{F}\left(\boldsymbol{y}^{\star}, \boldsymbol{z}^{(l)}\right) / N$. While $\quad 314$ such an approach is straightforward, it may be quite demanding from a numerical viewpoint, as 315 a large number of samples $N$ (proportional to $1 / p_{F}$ ) are required to ensure a sufficiently accurate 316 estimator. In order to reduce the number of simulations, one can resort to advanced variants of 317 Monte Carlo simulation, as discussed in the sequence. 318 The structure of eq. (5) indicates that the normalized response function is actually the maximum 319 among a series of individual functions which depend linearly on vector $\boldsymbol{z}$. Hence, the boundary 320 separating the realizations $\boldsymbol{z}$ that cause failure or not in the standard normal space is a collection $\quad 321$ 
of hyperplanes given by the limit state surface $r(\boldsymbol{y}, \boldsymbol{z})=1[66,67]$, as depicted schematically 322 in Figure 1. This fact brings important information which can be exploited for evaluating the 323 failure probability in eq. (7). For example, Directional Importance Sampling (DIS) [42, 43] is an 324 advanced simulation method that is built on such information and is capable of estimating small 325 failure probabilities with high accuracy and a reduced number of samples. As the application of 326 DIS is well documented $[42,43]$, only the essential aspects of this simulation method are described 327 in the following.

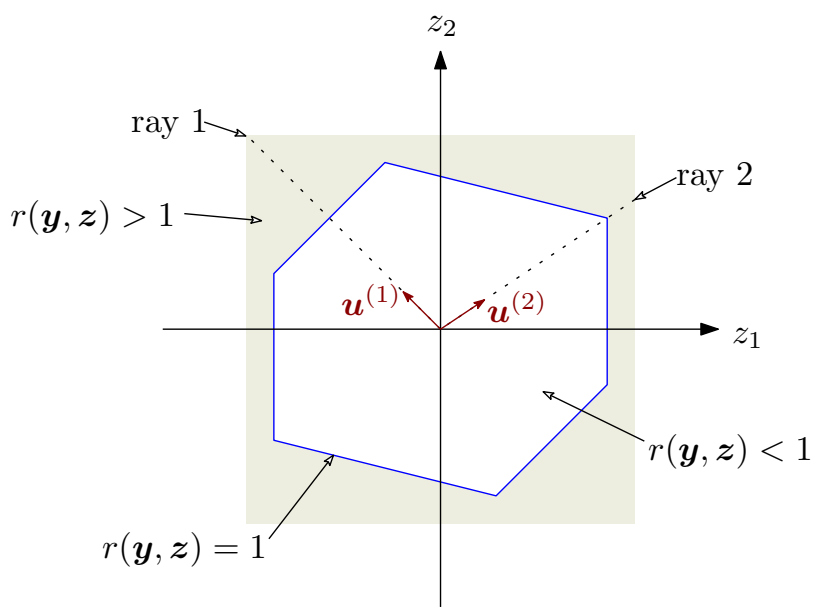

Figure 1: Schematic representation of failure domain associated with probability integral.

DIS is built upon two key concepts. The first one is that vector $\boldsymbol{z}$ associated with the stochastic 329 excitation is represented in a polar fashion. That is, $\boldsymbol{z}=R \boldsymbol{u}$, where $R$ is a scalar representing 330 the length of $\boldsymbol{z}$ (in terms of Euclidean norm) and $\boldsymbol{u}$ is a direction vector (with unit Euclidean 331 norm) pointing towards $\boldsymbol{z}$. The advantage of such representation is that all structural responses of 332 interest associated with realizations lying along the ray that originates at $\mathbf{0}$ and that points in the 333 direction of vector $\boldsymbol{u}$ can be calculated with a single system analysis, by proper scaling according 334 to the length $R$. Such property can be understood due to the linearity of the structural response 335 with respect to $\boldsymbol{z}$, as noted from eq. (4) and Appendix B. In other words, if the structural 336 response is calculated for a given realization $\boldsymbol{u}$ of the direction vector, the responses for all values 337 of $R \in(0, \infty)$ associated with that direction can be determined by simply amplifying by factor $R$. $\quad 338$ Such concept is illustrated schematically in Figure 1, where two direction vectors $\boldsymbol{u}^{(l)}, l=1,2$ are 339 illustrated as well as their corresponding rays. Provided that the structural response is evaluated 340 for each of those direction vectors, it is then possible to calculate the structural response at ${ }^{341}$ any point along the respective ray by amplification according to $R$. The second concept behind 342 
DIS is that samples of the direction vector $\boldsymbol{u}$ are generated with a specially design Importance 343 Sampling density function. Such density function ensures that those direction vectors which are 344 highly relevant for calculating the failure probability are sampled frequently. For example, from 345 the schematic representation in Figure 1, it is noted that the portion of the ray associated with 346 sample $\boldsymbol{u}^{(1)}$ that falls within the failure domain is longer than that associated with ray 2. Thus, 347 the Importance Sampling density function gives more importance to sampling direction $\boldsymbol{u}^{(1)}$ and ${ }_{348}$ less relevance to sampling direction $\boldsymbol{u}^{(2)}$. In order to ensure that all directions that cause failure 349 are properly accounted for, the Importance Sampling density function is constructed considering 350 that failure may take place at any time within the duration of the stochastic excitation as well as 351 for any of the responses of interest. This means that the Importance Sampling density function 352 comprises the occurrence of failure at $n_{T}$ times instants over $n_{R}$ responses. Numerical experience 353 as reported in [43] indicates that DIS is applicable to small and large scale structural models 354 and that it provides highly accurate estimates of the failure probability with a modest number of 355 simulations (in the order of few hundreds). 356

\subsection{Summary of proposed approach 357}

The proposed approach for minimizing the first excursion probability of a linear structure subject 358 to a stochastic Gaussian loading considering design variables belonging to a discrete set can be 359 summarized as follows. 360

1. Characterize the stochastic input load by means of the Karhunen-Loève expansion, as de- 361 scribed in eq. (1). 362

2. Select the structural responses to be controlled as well as their corresponding threshold 363 levels. 364

3. Define the set of discrete values $\Omega_{j}, j=1, \ldots, n_{y}$ that the design variables of the problem 365 $y_{j}, j=1, \ldots, n_{y}$ may assume. 366

4. Solve the (deterministic) optimization problem in eq. (12) by means of Genetic Algorithms 367 (see Section 3.2) and determine the optimal design $\boldsymbol{y}^{\star}$. Recall that for each candidate solution 368 evaluated by the optimization algorithm, it is necessary to carry out structural analysis in 369 order to assemble matrix $\boldsymbol{A}$ (see eq. (5) and Appendix B) and calculate the associated 370 operator norm (see eq. (11)). 371

5. Estimate the first excursion probability associated with the optimal design $\boldsymbol{y}^{\star}$ by means of 372 Directional Importance Sampling (see Section 3.3). 373 


\section{Examples}

4.1. Test Example 1: Single-degree-of-freedom oscillator subject to discrete white noise excitation 375 The first example consists of a single-degree-of-freedom oscillator subject to a stochastic ground 376 acceleration modeled as a discrete white noise, as depicted schematically in Figure 2. The objective 377 of the problem is selecting the value of the stiffness such that the first excursion probability of the 378 oscillator is minimized.



Figure 2: Example 1 - Single-degree-of-freedom oscillator subject to discrete white noise excitation.

The white noise excitation possesses a duration of $T=10[\mathrm{~s}]$, a spectral density $S=5 \times 10^{-4}{ }_{380}$ $\left[\mathrm{m}^{2} / \mathrm{s}^{3}\right]$ and is represented considering a time discretization of $\Delta t=0.01[\mathrm{~s}]$. Thus, the total 381 number of time steps is equal to $n_{T}=1001$. As the covariance matrix associated with a discrete 382 white noise process is of diagonal type, the Karhunen-Loève representation involves a total of 383 $n_{K L}=1001$ terms.

The oscillator possesses a mass $m=10^{4}[\mathrm{~kg}]$ and damping ratio $\zeta=5 \%$, while its stiffness $k$ is 385 a design variable to be selected within the discrete set $\Omega_{k}=\{1.0,1.1,1.2, \ldots, 4.9,5.0\}[\mathrm{MN} / \mathrm{m}]$. 386 The responses of interest are the relative displacement of the oscillator with respect to the ground 387 and the absolute acceleration (that is, $n_{R}=2$ ); the respective threshold levels are set as $b_{1}=0.01 \quad 388$ $[\mathrm{m}]$ for the displacement and $b_{2}=3\left[\mathrm{~m} / \mathrm{s}^{2}\right]$ for the acceleration. The probability that any of 389 these two responses exceed their respective threshold levels within $t \in[0,10][\mathrm{s}]$ is calculated using 390 Directional Importance Sampling, considering a total of $N=500$ samples of the stochastic ground 391 acceleration. It should be noted that although this example involves a relatively simple model, 392 the calculation of the associated failure probability is already quite challenging, as it comprises a 393 total of $n_{K L}=1001$ dimensions and $n_{R} n_{T}=2002$ responses to be controlled. 394 The RBO problem to be solved is the following in mathematical terms.

subject to:

$$
k \in \Omega_{k}=\{1.0,1.1,1.2, \ldots, 4.9,5.0\}[\mathrm{MN} / \mathrm{m}]
$$


As the discrete set $\Omega_{k}$ involves 41 elements, it is decided to assess the probability associated with 396 each of those possible designs. Hence, for this first example only, no optimization is considered. 397 Figure 3 contains the results associated with each element of set $\Omega_{k}$ : the top plot contains the 398 operator norm of matrix $\boldsymbol{A}$ as a function of the stiffness while the bottom plot contains the failure 399 probability as a function of $k$. It is observed that the sets of points associated with the operator 400 norm and failure probability possess a similar behavior: that is, first decreasing with respect to 401 the stiffness up to the point $k=3[\mathrm{MN} / \mathrm{m}]$; and then, increasing from that point onward. Such 402 similar behavior reinforces the concept of applying the operator norm theorem for replacing the ${ }_{403}$ RBO problem in eq. (8) with the deterministic problem in eq. (12), as both curves $\|\boldsymbol{A}(k)\|_{\infty, 2}$ vs. ${ }_{404}$ $k$ and $p_{F}(k)$ vs. $k$ reach their minimum for the same value $k=3[\mathrm{MN} / \mathrm{m}]$. $\quad 405$
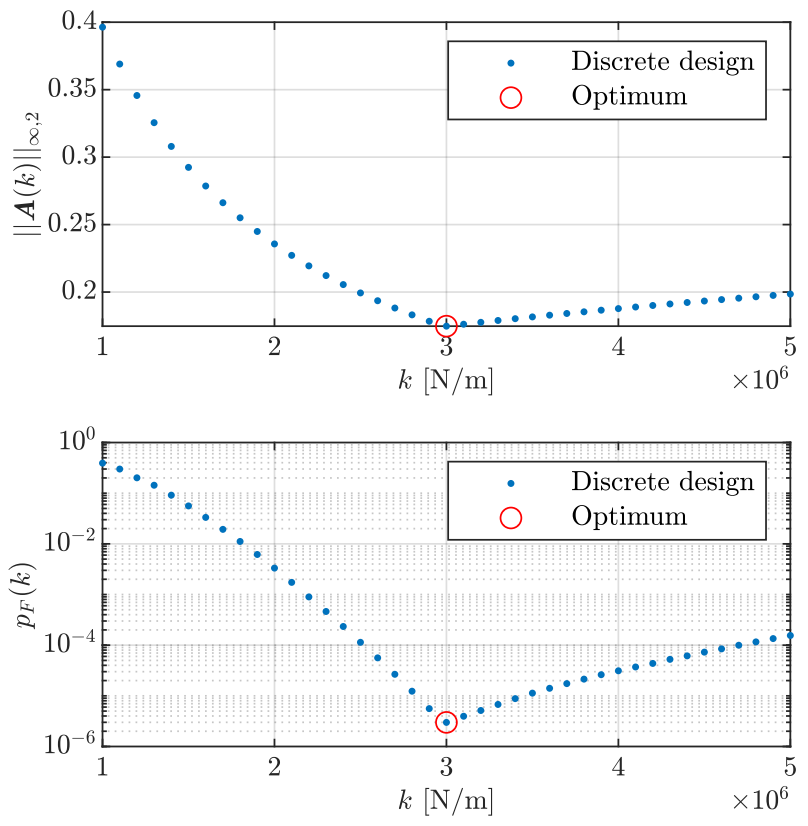

Figure 3: Example 1 - Operator norm $\left(\|\boldsymbol{A}(k)\|_{\infty, 2}\right)$ and failure probability $\left(p_{F}(k)\right)$ versus discrete-valued stiffness (k).

Figure 4 presents the failure probability $\left(p_{F}(k)\right)$ as a function of the operator norm of matrix $\boldsymbol{A} 406$ (that is, $\left.\|\boldsymbol{A}(k)\|_{\infty, 2}\right)$. For this set of points, it is observed that the minimum value of $\|\boldsymbol{A}(k)\|_{\infty, 2} \quad{ }_{407}$ corresponds to the minimum value of $p_{F}(k)$, as already noted from Figure 3. From a physical viewpoint, the results shown in the bottom plot of Figure 3 possess a relevant ${ }_{409}$ interpretation. For small stiffness values, the oscillator is quite flexible and thus, it is more likely 410 to fail because of the displacement condition rather than by the acceleration condition. As the ${ }_{411}$ stiffness increases (up to $3[\mathrm{MN} / \mathrm{m}]$ ), the oscillator is less flexible, thus decreasing its maximum ${ }_{412}$ 


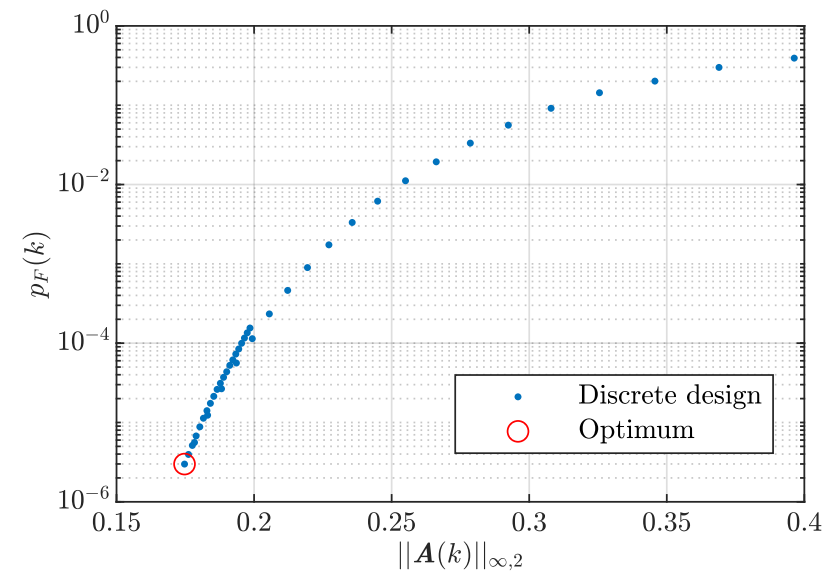

Figure 4: Example 1 - Operator norm $\left(\|\boldsymbol{A}(k)\|_{\infty, 2}\right)$ versus failure probability $\left(p_{F}(k)\right)$.

displacement and consequently, its failure probability. However, for stiffness values larger than 413 $3[\mathrm{MN} / \mathrm{m}]$, the oscillator becomes too stiff and the failure criterion associated with acceleration ${ }_{414}$ becomes more relevant, thus explaining the increase in failure probability. Such behavior had 415 already been observed within the context of RBO problems involving continuous design variables 416 in $[5]$.

\subsection{Test Example 2: Three-story shear frame subject to discrete white noise excitation}

The second example involves the three-story shear beam model subject to a stochastic ground 419 acceleration illustrated in Figure 5. The objective of the problem is selecting the cross sections of ${ }_{420}$ the columns of each floor such that the first excursion probability of the structure is minimized. ${ }^{421}$

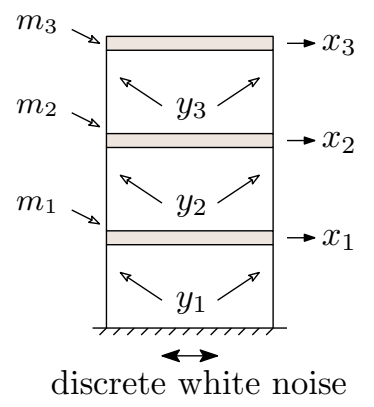

Figure 5: Example 2 - Three-story shear frame subject to discrete white noise excitation.

The ground acceleration is modeled by means of the same discrete white noise process considered ${ }_{422}$ in Section 4.1. Concerning the structural model, it is assumed that the mass of the model is ${ }_{423}$ concentrated at the infinitely stiff floor beams and is equal to $m_{i}=50 \times 10^{3}[\mathrm{~kg}], i=1,2,3$. A 424 classical modal damping of $\zeta_{i}=2 \%, i=1,2,3$ is considered for each mode. Each floor of the shear ${ }_{425}$ frame is supported by two identical, perfectly clamped steel columns, whose Young's modulus is ${ }_{426}$ 
equal to $E=2 \times 10^{11}[\mathrm{~N} / \mathrm{m}]$. The cross section of the columns of the three stories are the design ${ }^{427}$ variables of the problem, which must be selected from the database of HEM profiles belonging ${ }_{428}$ to the Euronorm 53-62. As the considered database comprises a total of 24 profiles and there ${ }_{429}$ are $n_{y}=3$ design variables, the set $\Omega$ of discrete elements for design comprises a total of 13824430 entries. Because of constructive reasons, it is imposed that the depth of the column's cross section 431 of floor $i+1$ should be equal or smaller than the depth of the column's cross section of floor $i . \quad 432$ Such condition imposes then $n_{C}=2$ deterministic constraints to the optimization problem. $\quad{ }_{433}$ The responses of interest of the system are the interstory drifts and the absolute acceleration 434 of each floor $\left(n_{R}=6\right)$, which should remain below prescribed thresholds of $7 \times 10^{-3}[\mathrm{~m}]$ and $5 \quad{ }_{435}$ $\left[\mathrm{m} / \mathrm{s}^{2}\right]$, respectively. The probability that any of these responses exceeds its prescribed threshold is 436 calculated by means of Directional Importance Sampling, considering a total of $N=500$ samples. $\quad{ }^{437}$ The RBO problem to be solved is: $\quad{ }_{438}$

$$
\begin{aligned}
& \min _{\boldsymbol{y}} p_{F}(\boldsymbol{y}) \\
& \text { subject to: } \\
& d_{p}\left(y_{l+1}\right)-d_{p}\left(y_{l}\right) \leq 0, l=1,2 \\
& y_{j} \in \Omega_{\mathrm{HEM}}, j=1,2,3
\end{aligned}
$$

where $d_{p}\left(y_{l}\right)$ denotes the cross section's depth associated with the $l$-th design variable; and $\Omega_{\mathrm{HEM}}{ }^{439}$ denotes the set of 24 HEM admissible steel profiles. 440 As a means for exploring the problem at hand in a preliminary fashion, a total of 1000 designs are ${ }_{441}$ generated at random (each of them containing three cross sections chosen from the HEM profile ${ }_{442}$ database). The operator norms and failure probabilities associated with each of these designs are ${ }_{443}$ shown in Figure 6 with blue dots. It is observed that these random designs span about three 444 orders of magnitude of failure probabilities, indicating that the performance of the three-story ${ }_{445}$ shear beam model is sensitive to the selection of the column's cross sections. Furthermore, the ${ }_{446}$ cloud of points suggests that finding the minimum value of the operator norm indeed corresponds 447 to finding the minimum value of the failure probability. 448 In a next step, the RBO problem is addressed by means of the decoupling approach proposed ${ }_{449}$ in this work. For this purpose, the optimization problem in eq. (12) is addressed by means of 450 Genetic Algorithms, considering a population size of 50 individuals. The optimum is found after 60451 


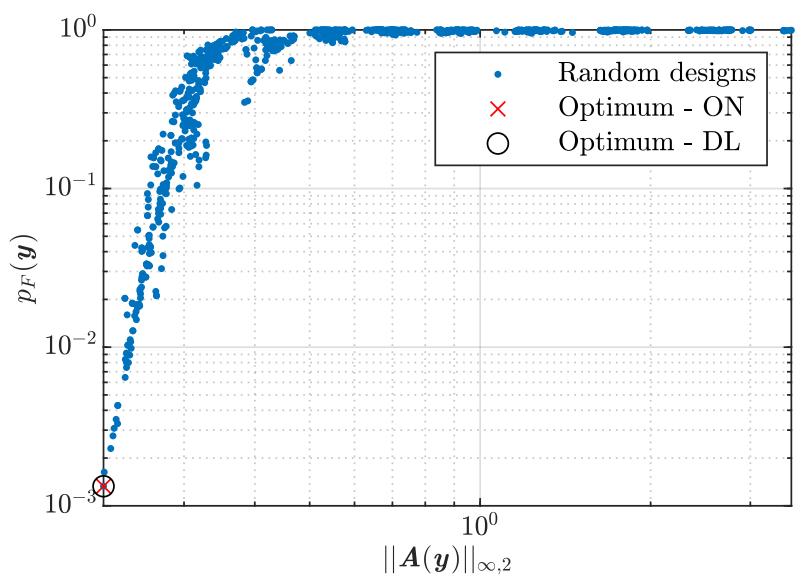

Figure 6: Example 2 - Operator norm $\left(\|\boldsymbol{A}(\boldsymbol{y})\|_{\infty, 2}\right)$ versus failure probability $\left(p_{F}(\boldsymbol{y})\right)$. ON: operator norm; DL: double loop.

generations and is equal to $\boldsymbol{y}^{\star}=[\text { HEM320, HEM300, HEM300 }]^{T}$. Then, a single reliability analysis ${ }_{452}$ is conducted for estimating the failure probability associated with that optimal design, and it is ${ }_{453}$ found that $p_{F}\left(\boldsymbol{y}^{\star}\right)=1.3 \times 10^{-3}$. The results obtained with such procedure are plotted in Figure ${ }^{454}$ 6 with a red x mark. In addition and as a means to validate the results described previously, the ${ }_{455}$ RBO problem is solved once more, this time using a classical double loop approach. This means ${ }_{456}$ that the optimization problem in eq. (8) is solved by means of Genetic Algorithms and that for ${ }^{457}$ each candidate design, the failure probability is evaluated by means of Directional Importance ${ }^{458}$ Sampling. The optimum design obtained with such procedure is shown with a black circle in ${ }^{459}$ Figure 6. As it can be noticed from that Figure, the results produced with both the proposed ${ }_{460}$ decoupling approach employing operator norm $(\mathrm{ON})$ and double loop (DL) are identical. That is, 461 for both cases the optimal design is $\boldsymbol{y}^{\star}=[\text { HEM320, HEM300, HEM300 }]^{T}$ with failure probability ${ }_{462}$ $p_{F}\left(\boldsymbol{y}^{\star}\right)=1.3 \times 10^{-3}$. However, the numerical efforts associated with each approach are quite ${ }_{463}$ different, as shown in Figure 7. This Figure illustrates the total execution times associated with ${ }^{464}$ the optimization considering the operator norm and the classical double loop approach. These ${ }_{465}$ execution times are normalized by the execution time associated with the operator norm. It is ${ }_{466}$ noted that the operator norm offers a significant reduction in computation time.

\subsection{Example 3: Ten-story moment frame subject to filtered white noise excitation 468}

The third example involves the ten-story moment frame of Figure 8, which is subjected to a 469 stochastic ground acceleration. The objective of the problem is selecting the cross sections of the ${ }^{470}$ columns of each floor such that the first excursion probability of the frame is minimized. ${ }_{471}$ The stochastic ground acceleration is characterized as a modulated white noise excitation, filtered ${ }_{472}$ 


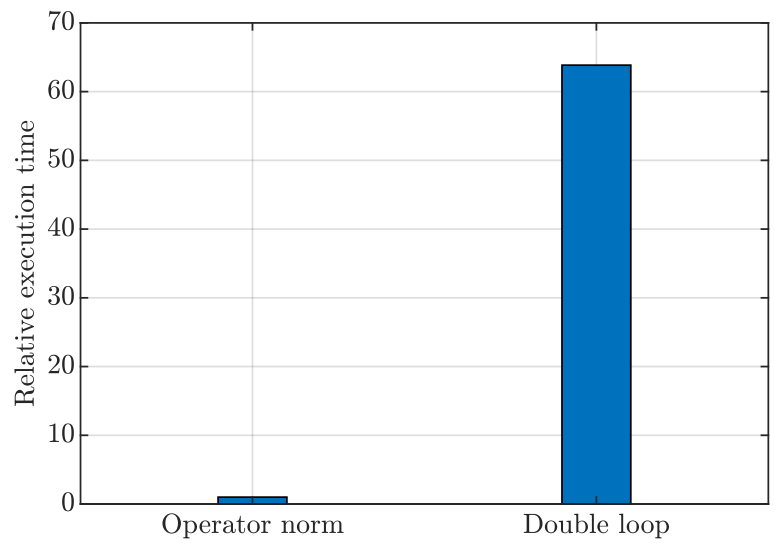

Figure 7: Example 2 - relative execution time.

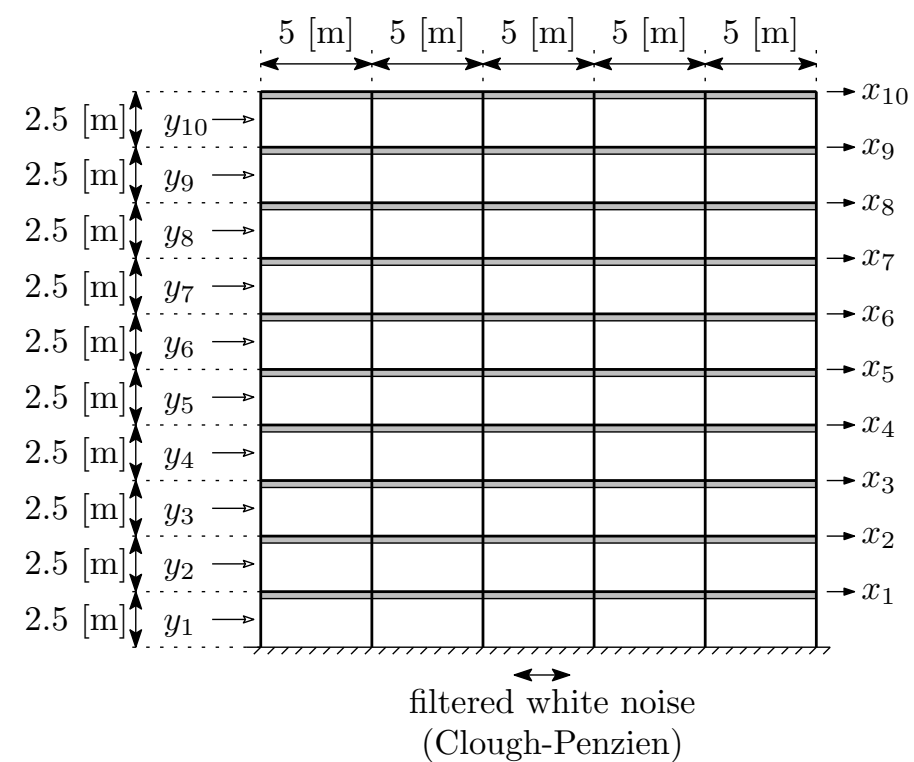

Figure 8: Example 3 - Ten-story moment frame subject to filtered white noise excitation.

by means of the Clough-Penzien model (see, e.g. [68]). The discrete white noise process possesses 473 a duration of $T=15[\mathrm{~s}]$, a spectral density $S=3 \times 10^{-4}\left[\mathrm{~m}^{2} / \mathrm{s}^{3}\right]$ and is represented considering a ${ }_{474}$ time discretization of $\Delta t=0.01[\mathrm{~s}]$. Thus, the total number of time steps is equal to $n_{T}=1501$. 475 This discrete white noise process is modulated by the following function $m(t)$ :

$$
m(t)= \begin{cases}t^{2} / 16 & \text { if } 0[\mathrm{~s}] \leq t \leq 4[\mathrm{~s}] \\ 1 & \text { if } 4[\mathrm{~s}]<t \leq 10[\mathrm{~s}] \\ e^{(t-10)^{2}} & \text { if } 10[\mathrm{~s}]<t \leq 15[\mathrm{~s}]\end{cases}
$$

The Clough-Penzien filter possesses circular natural frequencies $\omega_{g, 1}=15.6[\mathrm{rad} / \mathrm{s}]$ and $\omega_{g, 2}=1.0 \quad 477$ $[\mathrm{rad} / \mathrm{s}]$ and corresponding damping ratios $\zeta_{g, 1}=0.6$ and $\zeta_{g, 2}=0.9$. The stochastic process is ${ }_{478}$ 
represented by means of the Karhunen-Loève expansion considering a total of $n_{K L}=1501$ terms. $\quad 479$ The structural model considers the moment resistance provided by the columns and beams of the 480 structure plus the contribution of the reinforced concrete floor slab. It is assumed that the mass 481 of the model is concentrated at slab level and is equal to $m_{i}=100 \times 10^{3}[\mathrm{~kg}], i=1, \ldots, 10$. A 482 classical modal damping of $2 \%$ is considered for all modes. Each floor of the frame is supported 483 by six identical, perfectly clamped steel columns, whose Young's modulus is equal to $E=2 \times 10^{11}{ }_{484}$ $[\mathrm{N} / \mathrm{m}]$. The cross section of the columns of the ten stories are the design variables of the problem, 485 which must be selected from the database of HEM profiles belonging to the Euronorm 53-62. 486 Given that $n_{y}=10$ and that the HEM database contains 24 profiles, the set $\Omega$ possesses about ${ }^{487}$ $6 \times 10^{13}$ entries. Following an approach similar to the one in Section 4.2, it is imposed that the ${ }_{488}$ depth of the column's cross section of floor $i+1$ should be equal or smaller than the depth of 489 the column's cross section of floor $i$, leading to a total of $n_{C}=9$ deterministic constraints for the ${ }_{490}$ optimization problem. $\quad 491$ The responses of interest of the system are the interstory drifts and the absolute acceleration of 492 each floor $\left(n_{R}=20\right)$, which should remain below prescribed thresholds of $5 \times 10^{-3}[\mathrm{~m}]$ and $3{ }_{493}$ $\left[\mathrm{m} / \mathrm{s}^{2}\right]$, respectively. The probability that any of these responses exceeds its prescribed threshold 494 is calculated by means of Directional Importance Sampling, considering a total of $N=1000{ }_{495}$ $\begin{array}{ll}\text { samples. } & 496\end{array}$ The RBO problem to be solved is:.

$$
\begin{aligned}
& \min _{\boldsymbol{y}} p_{F}(\boldsymbol{y}) \\
& \text { subject to: } \\
& d_{p}\left(y_{l+1}\right)-d_{p}\left(y_{l}\right) \leq 0, l=1, \ldots, 9 \\
& y_{j} \in \Omega_{\mathrm{HEM}}, j=1, \ldots, 10
\end{aligned}
$$

where $d_{p}\left(y_{l}\right)$ and $\Omega_{\text {HEM }}$ have been defined previously. It should be noted that while the structural ${ }_{498}$ model associated with this RBO problem is relatively simple (that is, a 10-story moment frame), it 499 serves the purposes of this contribution. This point becomes clearer when analyzing the numerical 500 performance, as discussed at the end of this Section. 501 Before solving the optimization problem, 6400 random designs are explored. Each of these random 502 designs contains 10 HEM profiles. Figure 9 illustrates the values of the operator norm and failure 503 
probability associated with each of those designs, represented with blue dots. A pattern similar to 504 the one observed in in Section 4.2 is found in this Figure, that is, the random samples span a wide 505 range of failure probabilities and the minimum operator norm value coincides with the minimum 506 failure probability.

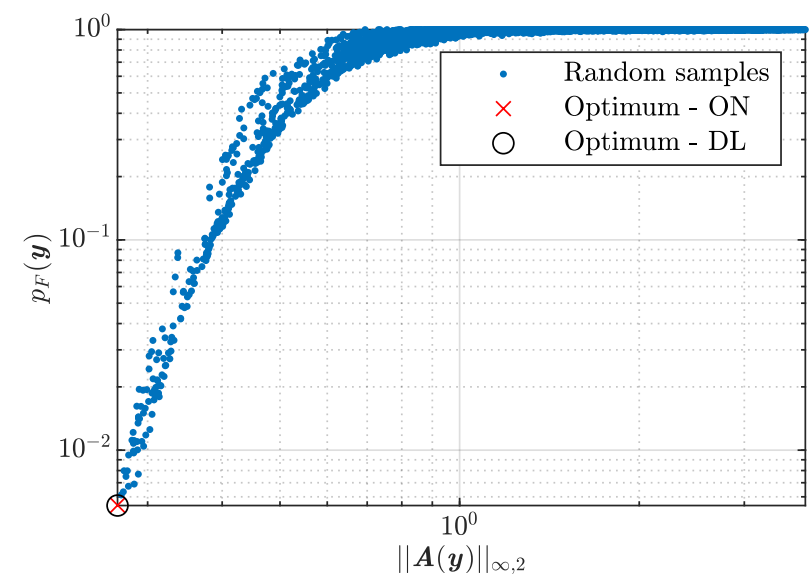

Figure 9: Example 3 - Operator norm $\left(\|\boldsymbol{A}(\boldsymbol{y})\|_{\infty, 2}\right)$ versus failure probability $\left(p_{F}(\boldsymbol{y})\right)$. ON: operator norm; DL: double loop.

After the preliminary analysis carried out by means of random samples, the problem of finding the 508 column's sections that minimize the first excursion probability associated with the 10 story frame 509 model is solved by means of Genetic Algorithms. For comparison purposes, both the classical 510 double loop approach and the decoupling approach based on operator norm are carried out. For 511 each case, a population size of 1280 individuals is considered in the implementation of Genetic 512 Algorithms. This number is larger than the one considered in Section 4.2 in order to cope with 513 the increased dimension of the design variable vector. The optimal designs found for each case 514 are reported in Table 1 in terms of the selected cross sections, the associated operator norms 515 and the failure probabilities; these optimal designs are also plotted in Figure 9 in terms of the 516 corresponding operator norm and failure probability. It is seen that while the optimum designs 517 found by each procedure are not exactly the same, they are quite similar and possess almost 518 identical values for the operator norm and failure probability. In this sense, it should be kept 519 in mind that the optimization problem itself is quite challenging and that Genetic Algorithms 520 corresponds to a stochastic search procedure, so it is not surprising that the optimum designs 521 found in each case are slightly different.

Figure 10 illustrates the execution time associated with the optimization considering decoupling 523 by means of operator norm and the classical double loop (normalized by the execution time of 524 


\begin{tabular}{|c|c|c|}
\hline & \multicolumn{2}{|c|}{ Optimal design } \\
\hline Floor & $\mathrm{ON}$ & $\mathrm{DL}$ \\
\hline 1 & HEM500 & HEM700 \\
\hline 2 & HEM450 & HEM450 \\
\hline 3 & HEM360 & HEM340 \\
\hline 4 & HEM340 & HEM340 \\
\hline 5 & HEM320 & HEM300 \\
\hline 6 & HEM300 & HEM300 \\
\hline 7 & HEM300 & HEM280 \\
\hline 8 & HEM300 & HEM260 \\
\hline 9 & HEM300 & HEM260 \\
\hline 10 & HEM260 & HEM260 \\
\hline$\left\|\boldsymbol{A}\left(\boldsymbol{y}^{\star}\right)\right\|_{\infty, 2}$ & 0.2672 & 0.2671 \\
\hline$p_{F}\left(\boldsymbol{y}^{\star}\right)$ & $5.47 \times 10^{-3}$ & $5.45 \times 10^{-3}$ \\
\hline
\end{tabular}

Table 1: Example 3 - Optimal designs found applying decoupling approach by means of operator norm (ON) and classical double loop (DL).

optimization considering decoupling). As already noticed from the example in Section 4.2, the 525 application of the proposed approach leads to a drastic reduction of computation times. As an 526 additional reference, all computations were performed on a server equipped with 512 GB of RAM ${ }_{527}$ and two 64-thread AMD EPYC $6701 \mathrm{CPU} @ 2.65 \mathrm{GHz}$. The solution of the RBO problem by 528 means of the operator norm framework took $30.5[\mathrm{~s}]$ while the classical double-loop demanded 529 2600 [s], thus highlighting the advantages of the proposed framework. This is quite remarkable, 530 particularly taking into account that the structural model involved in the problem is relatively 531 simple, as already stated above. In this sense, the numerical challenge of solving the problem with 532 a double loop comes into the exhaustive exploration of the design space which must be carried out 533 combined with the reliability analysis conducted at each design explored. Hence, it is expected 534 that the proposed decoupling approach may offer considerable benefits in problems involving large 535 structural models where, in addition, one may take advantage of substructuring, as discussed in, 536 e.g. [63].

\section{Conclusions and Outlook}

This contribution presents a decoupling approach based on operator norm theory for the solution 539 of a class of RBO problems, namely minimization of the first excursion of a linear structure sub- 540 ject to a stochastic loading that involves design variables belonging to a discrete set. The core of 541 the proposed approach lies in the concept of decoupling, as the solution of the RBO problem is 542 




Figure 10: Example 3 - relative execution time.

replaced by one deterministic optimization problem (which involves no uncertainty propagation 543 at all) followed by one reliability analysis (which does not involve any optimization). For practi- 544 cal implementation, this decoupling approach is applied in conjunction with Genetic Algorithms, 545 which guarantees that the space of available discrete designs is examined exhaustively. Numerical 546 examples as considered in this contribution show that the proposed approach is capable of deter- 547 mining the same optimal design as that obtained by means of a standard, double loop technique, 548 but with a reduction of computation times close to two orders of magnitude. 549 While the approach presented in this contribution shows an excellent performance, it should be 550 kept in mind that as of yet, its range of application is quite specific: minimization of the failure 551 probability of a linear stochastic system with discrete design variables. Hence, future research 552 efforts will aim at extending the range of application considering, for example, weight minimiza- 553 tion under reliability constraints and risk-based optimization of linear stochastic systems. It 554 is envisioned that such extension could be achieved by relating the operator norm and failure 555 probability by means of an appropriate surrogate model. Another possible extension of the pro- 556 posed approach consists of extending its range of applications to RBO problems involving mixed 557 discrete-continuous design variables and non-Gaussian stochastic load. Yet another path for future 558 development involves the extension of the approach to the nonlinear case. However, this last goal 559 seems more challenging, as the linearity of the response with respect to the stochastic excitation 560 does no longer hold. Probably, methods based on equivalent linearization may be of help in such 561 situation. 


\section{Acknowledgments}

Matthias Faes gratefully acknowledges the financial support of the Research Foundation Flanders 564 (FWO) under grant number 12P3519N as well as the Alexander von Humboldt foundation. Marcos 565 Valdebenito acknowledges the support of ANID (National Agency for Research and Development, 566 Chile) under its program FONDECYT, grant number 1180271.

\section{Appendix A. Unit impulse response function}

Unit impulse response functions associated with different responses of interest can be conveniently 569 calculated by means of modal analysis. For example, under the assumption that the $i$-th response 570 of interest $\eta_{i}$ is a linear combination of the displacement vector $\boldsymbol{x}$, it can be shown that the unit 571 impulse response function $h_{i}(t)$ is given by (see, e.g. [49]):

$$
h_{i}(t, \boldsymbol{y})=\sum_{v=1}^{n_{D}} \frac{\alpha_{i, v}(\boldsymbol{y}) e^{-\zeta_{v}(\boldsymbol{y}) \omega_{v}(\boldsymbol{y}) t}}{\omega_{d, v}(\boldsymbol{y})} \sin \left(\omega_{d, v}(\boldsymbol{y}) t\right), i=1, \ldots, n_{R}
$$

where $\omega_{v}, v=1, \ldots, n_{D}$ are the natural frequencies; $\zeta_{v}, v=1, \ldots, n_{D}$ are the damping ratios; 573

$\omega_{d, v}=\omega_{v} \sqrt{\left(1-\zeta_{v}^{2}\right)}, v=1, \ldots, n_{D}$ are the damped frequencies; $\alpha_{i, v}, i=1, \ldots, n_{R}, v=1, \ldots, n_{D} \quad{ }^{574}$ are the modal participation factors, defined as:

$$
\alpha_{i, v}(\boldsymbol{y})=\frac{\boldsymbol{q}_{i}^{T} \boldsymbol{\phi}_{v}(\boldsymbol{y}) \phi_{v}(\boldsymbol{y})^{T} \boldsymbol{\rho}}{\boldsymbol{\phi}_{v}(\boldsymbol{y})^{T} \boldsymbol{M}(\boldsymbol{y}) \boldsymbol{\phi}_{v}(\boldsymbol{y})}, i=1, \ldots, n_{R}, v=1, \ldots, n_{D}
$$

in addition, $\phi_{v}, v=1, \ldots, n_{D}$ are the eigenvectors associated with the eigenproblem of the un- 576 damped equation of motion; and $\boldsymbol{q}_{i}$ is a vector such that $\eta_{i}=\boldsymbol{q}_{i}^{T} \boldsymbol{x}$. The contribution of higher 577 order modes to the unit impulse response function in eq. (A.1) can be neglected for several cases 578 of practical interest [49].

The expression in eq. (A.1) presents a formula for calculating an impulse response function as- 580 sociated with displacements. In case the response of interest is a velocity or acceleration, the 581 associated unit impulse response function is expressed in terms of the corresponding time deriva- 582 
tive of eq. (A.1), that is:

$$
\begin{array}{r}
\frac{\partial h_{i}(t, \boldsymbol{y})}{\partial t}=\sum_{v=1}^{n_{D}} \frac{\alpha_{i, v}(\boldsymbol{y}) e^{-\zeta_{v}(\boldsymbol{y}) \omega_{v}(\boldsymbol{y}) t}}{\omega_{d, v}(\boldsymbol{y})}\left(\omega_{d, v}(\boldsymbol{y}) \cos \left(\omega_{d, v}(\boldsymbol{y}) t\right)-\zeta_{v}(\boldsymbol{y}) \omega_{v}(\boldsymbol{y}) \sin \left(\omega_{d, v}(\boldsymbol{y}) t\right)\right) \\
i=1, \ldots, n_{R} \\
\frac{\partial^{2} h_{i}(t, \boldsymbol{y})}{\partial t^{2}}=\sum_{v=1}^{n_{D}} \frac{\alpha_{i, v}(\boldsymbol{y}) e^{-\zeta_{v}(\boldsymbol{y}) \omega_{v}(\boldsymbol{y}) t}}{\omega_{d, v}(\boldsymbol{y})}\left(-2 \zeta_{v}(\boldsymbol{y}) \omega_{v}(\boldsymbol{y}) \omega_{d, v}(\boldsymbol{y}) \cos \left(\omega_{d, v}(\boldsymbol{y}) t\right)+\right. \\
\left.\zeta_{v}(\boldsymbol{y})^{2} \omega_{v}(\boldsymbol{y})^{2} \sin \left(\omega_{d, v}(\boldsymbol{y}) t\right)-\omega_{d, v}(\boldsymbol{y})^{2} \sin \left(\omega_{d, v}(\boldsymbol{y}) t\right)\right), i=1, \ldots, n_{R}
\end{array}
$$

\section{Appendix B. Discretization of convolution integral}

The convolution integral in eq. (3) can be approximated taking into account the discrete repre- 585 sentation of the loading in eq. (1). Thus, the $i$-th response at time instant $t_{k}$ is equal to:

$$
\begin{aligned}
\eta_{i}\left(t_{k}, \boldsymbol{y}, \boldsymbol{z}\right) & =\sum_{l_{1}=1}^{k} \epsilon_{l_{1}} h_{i}\left(\boldsymbol{y}, t_{k}-t_{l_{1}}\right) f\left(t_{l_{1}}, \boldsymbol{z}\right) \Delta t \\
& =\sum_{l_{1}=1}^{k} \epsilon_{l_{1}} h_{i}\left(t_{k}-t_{l_{1}}\right)\left(\sum_{l_{2}=1}^{n_{K L}} \psi_{l_{1}, l_{2}} \sqrt{\lambda_{l_{2}}} z_{l_{2}}\right) \Delta t \\
& =\boldsymbol{a}_{i_{k}}(\boldsymbol{y}) \boldsymbol{z}, i=1, \ldots, n_{\eta}, k=1, \ldots, n_{T}
\end{aligned}
$$

where $\psi_{l_{1}, l_{2}}$ is the $\left(l_{1}, l_{2}\right)$-th element of matrix $\boldsymbol{\Psi} ; \epsilon_{l_{1}}$ is a coefficient depending on the quadrature 587 scheme used to approximate the convolution integral; and $\boldsymbol{a}_{i_{k}}$ is a row vector of dimension $n_{K L} \times 1 \quad{ }_{588}$ defined as:

$$
\begin{array}{r}
\boldsymbol{a}_{i_{k}}(\boldsymbol{y})=\left[\sum_{l_{1}=1}^{k} \Delta t \epsilon_{l_{1}} h_{i}\left(\boldsymbol{y}, t_{k}-t_{l_{1}}\right) \psi_{l_{1}, 1} \sqrt{\lambda_{1}}, \ldots, \sum_{l_{1}=1}^{k} \Delta t \epsilon_{l_{1}} h_{i}\left(\boldsymbol{y}, t_{k}-t_{l_{1}}\right) \psi_{l_{1}, n_{K L}} \sqrt{\lambda_{n_{K L}}}\right] \\
i_{k}=(i-1) n_{T}+k, i=1, \ldots, n_{R}, k=1, \ldots, n_{T}
\end{array}
$$

It is straightforward to verify that matrix $\boldsymbol{A}_{i}$ in eq. (4) collects all row vectors $\boldsymbol{a}_{i_{k}}$ associated with 590 the $i$-th response, that is:

$$
\boldsymbol{A}_{i}(\boldsymbol{y})=\left[\begin{array}{c}
\boldsymbol{a}_{i_{1}}(\boldsymbol{y}) \\
\vdots \\
\boldsymbol{a}_{i_{n_{T}}}(\boldsymbol{y})
\end{array}\right], i=1, \ldots, n_{R}
$$


In this work, the trapezoidal integration rule is chosen for approximating the convolution integral 592 (see, e.g. [69]). Thus, $\epsilon_{l_{1}}$ is selected as $\epsilon_{l_{1}}=1 / 2$ if $l_{1}=1$ or $l_{1}=k$; otherwise, $\epsilon_{l_{1}}=1$. 593

\section{References}

[1] G. Schuëller, H. Jensen, Computational methods in optimization considering uncertainties 595 - An Overview, Computer Methods in Applied Mechanics and Engineering 198 (1) (2008) 596 2-13. doi:10.1016/j.cma.2008.05.004.

[2] M. Valdebenito, G. Schuëller, A survey on approaches for reliability-based optimization, 598 Structural and Multidisciplinary Optimization 42 (5) (2010) 645-663.

[3] A. Carlon, R. Lopez, L. Espath, L. Miguel, A. Beck, A stochastic gradient approach for the 600 reliability maximization of passively controlled structures, Engineering Structures 186 (2019) 601 1 - 12. doi:https://doi.org/10.1016/j.engstruct.2019.01.121. 602 URL http://www.sciencedirect.com/science/article/pii/S0141029618328402 603

[4] H. Jensen, M. Valdebenito, G. Schuëller, D. Kusanovic, Reliability-based optimization of 604 stochastic systems using line search, Computer Methods in Applied Mechanics and Engineer- 605 ing 198 (49-52) (2009) 3915-3924.

[5] H. Jensen, D. Jerez, M. Valdebenito, An adaptive scheme for reliability-based global design 607 optimization: A Markov chain Monte Carlo approach, Mechanical Systems and Signal Pro- 608 cessing 143 (2020) 106836. doi:https://doi.org/10.1016/j.ymssp.2020.106836. 609 URL http://www.sciencedirect.com/science/article/pii/S0888327020302223 610

[6] A. Taflanidis, J. Beck, An efficient framework for optimal robust stochastic system design us- ${ }^{611}$ ing stochastic simulation, Computer Methods in Applied Mechanics and Engineering 198 (1) 612 (2008) 88-101.

[7] J. Wang, L. Katafygiotis, Reliability-based optimal design of linear struc- ${ }^{614}$ tures subjected to stochastic excitations, Structural Safety 47 (2014) 29-38. 615 doi:http://dx.doi.org/10.1016/j.strusafe.2013.11.002. 616 URL http://www.sciencedirect.com/science/article/pii/S0167473013000854 617 
[8] J. Ching, S. Au, J. Beck, Reliability estimation for dynamical systems subject to stochastic 618 excitation using subset simulation with splitting, Computer Methods in Applied Mechanics 619 and Engineering 194 (12-16) (2005) 1557-1579.

[9] R. Foschi, H. Li, J. Zhang, Reliability and performance-based design: a computational ap- ${ }_{621}$ proach and applications, Structural Safety 24 (2-4) (2002) 205-218. 622

[10] M. Papadrakakis, N. Lagaros, Reliability-based structural optimization using neural networks ${ }_{623}$ and Monte Carlo simulation, Computer Methods in Applied Mechanics and Engineering 624 191 (32) (2002) 3491-3507. 625

[11] A. Suksuwan, S. Spence, A reliability model for the rapid optimization of large-scale ${ }_{626}$ and uncertain wind excited structures, Engineering Structures 190 (2019) 493 - 505. 627 doi:https://doi.org/10.1016/j.engstruct.2019.04.024. 628 URL http://www.sciencedirect.com/science/article/pii/S0141029618332127 ${ }_{22}$

[12] X. Chen, T. Hasselman, D. Neill, Reliability-based structural design optimization for prac- ${ }^{630}$ tical applications, in: Proceedings of the 38th AIAA Structures, Structural Dynamics, and ${ }_{631}$ Materials Conference, Florida, 1997.

[13] X. Du, W. Chen, Sequential optimization and reliability assessment method for efficient ${ }_{633}$ probabilistic design, Journal of Mechanical Design 126 (2) (2004) 225-233. 634

[14] I. Kaymaz, K. Marti, Reliability-based design optimization for elastoplastic mechanical struc- ${ }_{635}$ tures, Computers \& Structures 85 (10) (2007) 615-625. ${ }_{636}$

[15] N. Kuschel, R. Rackwitz, Two basic problems in reliability-based structural optimization, ${ }^{637}$ Mathematical Methods of Operations Research 46 (3) (1997) 309-333. 638

[16] J. Liang, Z. Mourelatos, J. Tu, A single-loop method for reliability-based design optimisation, ${ }_{639}$ International Journal of Product Development 5 (1-2) (2008) 76-92. 640

[17] Y. Aoues, A. Chateauneuf, Benchmark study of numerical methods for reliability-based design ${ }_{641}$ optimization, Structural and Multidisciplinary Optimization 41 (2) (2010) 277-294. 642

[18] I. Enevoldsen, J. Sørensen, Reliability-based optimization in structural engineering, Struc- 643 tural Safety 15 (3) (1994) 169-196. 
[19] G. Olsen, G. Vanderplaats, Method for nonlinear optimization with discrete design variables, ${ }^{645}$ AIAA Journal 27 (11) (1989) 1584-1589.

[20] M.-W. Huang, J. Arora, Optimal design with discrete variables: Some numerical experiments, ${ }_{647}$ International Journal for Numerical Methods in Engineering 40 (1) (1997) 165-188. ${ }_{648}^{6}$

[21] B. Blachowski, W. Gutkowski, Graph based discrete optimization in structural dynamics, 649 Bulletin of the Polish Academy of Sciences. Technical Sciences 62 (1) (2014). 650

[22] M. Cantoni, M. Marseguerra, E. Zio, Genetic Algorithms and Monte Carlo simulation for ${ }^{651}$ optimal plant design, Reliability Engineering \& System Safety 68 (1) (2000) 29-38. 652

[23] S. Gunawan, P. Papalambros, Reliability optimization with mixed continuous-discrete ran- ${ }_{653}$ dom variables and parameters, Journal of Mechanical Design 129 (2) (2007) 158-165. ${ }_{654}$

[24] R. Hassan, W. Crossley, Spacecraft reliability-based design optimization under uncertainty 655 including discrete variables, Journal of Spacecraft and Rockets 45 (2) (2008) 394-405. 656

[25] R. Stocki, K. Kolanek, S. Jendo, M. Kleiber, Study on discrete optimization techniques in 657 reliability-based optimization of truss structures, Computers \& Structures 79 (22-25) (2001) 658 $2235-2247$.

[26] B. Tolson, H. Maier, A. Simpson, B. Lence, Genetic Algorithms for reliability-based optimiza- 660 tion of water distribution systems, Journal of Water Resources Planning and Management ${ }_{661}$ $130(1)(2004) 63-72.662$

[27] J. Müller, C. Shoemaker, R. Piché, SO-MI: A surrogate model algorithm for computationally ${ }_{663}$ expensive nonlinear mixed-integer black-box global optimization problems, Computers \& Op- ${ }_{664}$ erations Research 40 (5) (2013) 1383 - 1400. doi:https://doi.org/10.1016/j.cor.2012.08.022. ${ }_{665}$ URL http://www.sciencedirect.com/science/article/pii/S0305054812001967 666

[28] M. Papadrakakis, N. Lagaros, V. Plevris, Design optimization of steel structures considering 667 uncertainties, Engineering Structures 27 (9) (2005) 1408-1418. 668

[29] S. Abspoel, L. Etman, J. Vervoort, R. van Rooij, A. Schoofs, J. Rooda, Simulation based ${ }_{669}$ optimization of stochastic systems with integer design variables by sequential multipoint 670 linear approximation, Structural and Multidisciplinary Optimization 22 (2) (2001) 125-138. 671 
[30] H. Jensen, M. Beer, Discrete-continuous variable structural optimization of systems under 672 stochastic loading, Structural Safety 32 (5) (2010) 293-304. 673

[31] H. Jensen, J. Sepulveda, Structural optimization of uncertain dynamical systems considering 674 mixed-design variables, Probabilistic Engineering Mechanics 26 (2) (2011) 269-280. 675

[32] H. Loh, P. Papalambros, A sequential linearization approach for solving mixed-discrete non- 676 linear design optimization problems, Tech. Rep. UM-MEAM-89-08, Univ. of Michigan, Ann 677 $\begin{array}{ll}\text { Arbor (1989). } & 678\end{array}$

[33] A. Suksuwan, S. Spence, Performance-based design optimization of uncertain wind ex- 679 cited systems under system-level loss constraints, Structural Safety 80 (2019) 13 - 31. 680 doi:https://doi.org/10.1016/j.strusafe.2019.03.004. 681 URL http://www.sciencedirect.com/science/article/pii/S0167473018301991 682

[34] M. Valdebenito, G. Schuëller, Reliability-based optimization considering design variables of ${ }_{683}$ discrete size, Engineering Structures 32 (9) (2010) 2919-2930. 684

[35] R. Lopez, A. Torii, L. Miguel, J. Sourza Cursi, An approach for the global reliability based ${ }_{685}$ optimization of the size and shape of truss structures, Mechanics \& Industry 16 (6) (2015) 686 603. doi:10.1051/meca/2015029. URL https://doi.org/10.1051/meca/2015029 688

[36] M. McDonald, S. Mahadevan, Reliability-based optimization with discrete and con- 689 tinuous decision and random variables, Journal of Mechanical Design 130 (6), 690 061401 (04 2008). arXiv:https://asmedigitalcollection.asme.org/mechanicaldesign/article- ${ }^{6} 1$ pdf/130/6/061401/5500636/061401_1.pdf, doi:10.1115/1.2898873. 692 URL https://doi.org/10.1115/1.2898873 693

[37] J. Tropp, Topics in sparse approximation, Ph.D. thesis, The University of Texas at Austin ${ }_{694}$ $(2004)$.

[38] M. Faes, M. Valdebenito, D. Moens, M. Beer, Bounding the first excursion probability of linear ${ }_{696}$ structures subjected to imprecise stochastic loading, Computers \& Structures 239 (2020) 697 106320. doi:https://doi.org/10.1016/j.compstruc.2020.106320. 698 URL http://www.sciencedirect.com/science/article/pii/S0045794920301231 699 
[39] M. G. Faes, M. A. Valdebenito, Fully decoupled reliability-based design optimization of struc- 700 tural systems subject to uncertain loads, Computer Methods in Applied Mechanics and En- 701 gineering 371 (2020) 113313. doi:https://doi.org/10.1016/j.cma.2020.113313. 702 URL http://www.sciencedirect.com/science/article/pii/S0045782520304989 703

[40] D. Goldberg, Genetic Algorithms in Search, Optimization, and Machine Learning, Addison 704 $\begin{array}{ll}\text { Wesley, Reading, MA, } 1989 . & 705\end{array}$

[41] M. Huang, C. Hsieh, J. Arora, A genetic algorithm for sequencing type problems in engi- 706 neering design, International Journal for Numerical Methods in Engineering 40 (17) (1997) 707 $3105-3115$.

[42] O. Ditlevsen, P. Bjerager, R. Olesen, A. Hasofer, Directional simulation in 709 Gaussian processes, Probabilistic Engineering Mechanics 3 (4) (1988) 207 - 217.710 doi:https://doi.org/10.1016/0266-8920(88)90013-6. 711 URL http://www.sciencedirect.com/science/article/pii/0266892088900136 712

[43] M. Misraji, M. Valdebenito, H. Jensen, C. Mayorga, Application of directional importance 713 sampling for estimation of first excursion probabilities of linear structural systems subject to 714 stochastic Gaussian loading, Mechanical Systems and Signal Processing 139 (2020) 106621. 715 doi:https://doi.org/10.1016/j.ymssp.2020.106621. 716 URL http://www.sciencedirect.com/science/article/pii/S0888327020300078 717

[44] L. Katafygiotis, J. Wang, Reliability analysis of wind-excited structures using domain decom- 718 position method and line sampling, Journal of Structural Engineering and Mechanics 32 (1) 719 (2009) $37-51$.

[45] G. Deodatis, Non-stationary stochastic vector processes: seismic ground mo- 721 tion applications, Probabilistic Engineering Mechanics 11 (3) (1996) 149 - 167.722 doi:https://doi.org/10.1016/0266-8920(96)00007-0. ${ }^{723}$ URL http://www.sciencedirect.com/science/article/pii/0266892096000070 724

[46] G. Stefanou, The stochastic finite element method: Past, present and future, Computer 725 Methods in Applied Mechanics and Engineering 198 (9-12) (2009) 1031-1051. 726 
[47] T. Soong, M. Grigoriu, Random Vibration of Mechanical and Structural Systems, Prentice ${ }^{727}$ Hall, Englewood Cliffs, New Jersey, 1993.

[48] K. Bathe, Finite Element Procedures, Prentice Hall, New Jersey, 1996.

[49] A. Chopra, Dynamics of structures: theory and applications to earthquake engineering, Pren- 730 tice Hall, 1995.

[50] S. Au, J. Beck, Estimation of small failure probabilities in high dimensions by subset simu- 732 lation, Probabilistic Engineering Mechanics 16 (4) (2001) 263-277. 733

[51] G. Schuëller, H. Pradlwarter, Benchmark study on reliability estimation in higher dimensions 734 of structural systems - An overview, Structural Safety 29 (2007) 167-182. 735

[52] D. Frangopol, Structural optimization using reliability concepts, Journal of Structural Engi- 736 neering 111 (11) (1985) 2288-2301.

[53] A. Taflanidis, J. Beck, Stochastic subset optimization for optimal reliability problems, Prob- 738 abilistic Engineering Mechanics 23 (2-3) (2008) 324-338. 739

[54] M. Valdebenito, G. Schuëller, Design of maintenance schedules for fatigue-prone metallic 740 components using reliability-based optimization, Computer Methods in Applied Mechanics 741 $\begin{array}{ll}\text { Engineering } 199 \text { (33-36) (2010) 2305-2318. } & 742\end{array}$

[55] A. Beck, W. Gomes, A comparison of deterministic, reliability-based and risk-based struc- 743 tural optimization under uncertainty, Probabilistic Engineering Mechanics 28 (2012) 18-29. 744 $\begin{array}{ll}\text { doi:10.1016/j.probengmech.2011.08.007. } & 745\end{array}$

[56] M.-W. Huang, J. Arora, Optimal design of steel structures using standard sections, Structural 746 and Muldisciplinary Optimization 14 (1) (1997) 24-35. 747

[57] J. Tomlin, Branch and bound methods for integer and non-convex programming, in: J.Abadie 748 (Ed.), lnteger and non-linear programming, North Holland, Amsterdam, 1970, pp. 437-450. 749

[58] W. Bennage, A. Dhingra, Single and multiobjective structural optimization in discrete- 750 continuous variables using simulated annealing, International Journal for Numerical Methods 751 in Engineering 38 (16) (1995) 2753-2773. 
[59] H.-G. Beyer, H.-P. Schwefel, Evolution strategies - A comprehensive introduction, Natural 753 Computing 1 (1) (2002) 3-52.

[60] M. Mitchell, An introduction to Genetic Algorithms, MIT Press, 1998.

[61] D. Beasley, D. Bull, R. Martin, An overview of Genetic Algorithms: Part 1, fundamentals, 756 University Computing 15 (2) (1993) 58-69.

[62] K. Deb, An efficient constraint handling method for genetic algorithms, Com- 758 puter Methods in Applied Mechanics and Engineering 186 (2) (2000) 311 - 338. 759 doi:https://doi.org/10.1016/S0045-7825(99)00389-8. 760 URL http://www.sciencedirect.com/science/article/pii/S0045782599003898 761

[63] H. Jensen, C. Papadimitriou, Sub-structure Coupling for Dynamic Analysis: Application to 762 Complex Simulation-Based Problems Involving Uncertainty, Springer Nature Switzerland AG 763 2019, 2019. doi:10.1007/978-3-030-12819-7.

[64] P. Umesha, M. Venuraju, D. Hartmann, K. Leimbach, Optimal design of truss structures 765 using parallel computing, Structural and Multidisciplinary Optimization 29 (4) (2005) 285- 766 297.

[65] G. Fishman, Monte Carlo: concepts, algorithms and applications, Springer, New York, NY, 768 1996.

[66] S. Au, J. Beck, First excursion probabilities for linear systems by very efficient importance 770 sampling, Probabilistic Engineering Mechanics 16 (3) (2001) 193-207. 771

[67] A. Der Kiureghian, The geometry of random vibrations and solutions by FORM and SORM, 772 Probabilistic Engineering Mechanics 15 (1) (2000) 81-90.

[68] A. Zerva, Spatial Variation of Seismic Ground Motions - Modeling and Engineering Appli- 774 cations, CRC Press, 2009.

[69] W. Gautschi, Numerical Analysis, 2nd Edition, Birkhäuser Boston, 2012. doi:10.1007/978-0- 776 8176-8259-0. 